\title{
La Conférence générale de l'UNESCO à Belgrade et la position de la Suisse. Quelques réfléxions.
}

Die UNESCO-Generalkonferenz in Belgrad und die Position der Schweiz. Einige Bemerkungen.

Philippe Grandjean

\section{(2) OpenEdition}

\section{Journals}

Édition électronique

URL : http://journals.openedition.org/aspd/1094

DOI : $10.4000 /$ aspd. 1094

ISSN : 1663-9669

Éditeur

Institut de hautes études internationales et du développement

Édition imprimée

Date de publication : 1 janvier 1981

Pagination : 253-259

ISSN : 1660-5934

Référence électronique

Philippe Grandjean, «La Conférence générale de I'UNESCO à Belgrade et la position de la Suisse. Quelques réfléxions. », Annuaire suisse de politique de développement [En ligne], 1 | 1981, mis en ligne le 18 janvier 2013, consulté le 08 septembre 2020. URL : http://journals.openedition.org/aspd/1094 ; DOI : https://doi.org/10.4000/aspd.1094 


\title{
LA CONFERENCE GENERALE DE L'UNESCO A BELGRADE ET LA POSITION DE LA SUISSE. QUELQUES REFLEXIONS
}

\author{
Philippe Grandjean
}

\section{DIE UNESCO-GENERALKONFERENZ IN BELGRAD UND DIE POSITION DER SCHWEIZ. EINIGE BEMERKUNGEN}

\section{Zusammenfassung}

Die 21. UNESCO-Generalkonferenz beschloss u.a. die Errichtung einer Neuen Internationalen Informations- und Kommunikationsordnung (NIIO).

Die Haltund der Schweiz entsprach der klassischen Haltung gegenober den Medien. Ihre Bevorzugung konkreter Projekte entspricht der offiziellen Konzeption der Entwicklungszusammenarbeit. Der Mac Bride Bericht spricht aber von einer generellen Domination der Industrielunder im Bereich des Informationswesens. Das Schweizer Konzept verdeckt die Ungleichgewichte im Gegenwärtigen internationalen Informationssystem.

Die Absichten des Mac Bride Berichts und der Mediendeklaration von Belgrad sind for den westlichen Verleger und Journalisten nicht zu akzeptieren. Sie zeigen den grossen Gegensatz zwischen einem liberalen Informationswesen un einer davon abweichenden Informationsauffassung. Die Schweiz wird eine Presse und Informationsfreiheit, die sie för sich geschaffen hat, international nicht in Frage stellen wollen. Wenn sich die Schweiz aber gegen die NIIO wehrt, so vor allem, weil sie die dominierende Position gefahrdet sieht. Wenn die dritte Welt eine ausgewogenere NIIO fordert, sieht die Schweiz die Gefahr einer Staatskontrolle Uber die Information.

Es stimmt zwar, dass, je zentralisierter eine Staatsmacht ist, desto kleiner ist der Spielraum der freien Presse. Man muss jedoch davon ausgehen, dass gerade die Entwicklungslander oft nach diesem Modell organisiert sind. Unsere Sicht der Medien wöre international jener, welche in den Entwicklungslandern verherrscht, an zupassen. Die Schweiz ist aber nur bereit, Infrastrukturverbesserungen im Informationswesen vorzunehmen, nicht aber, die Spielregeln zu undern. 
La 2 le Conférence générale de I'UNESCO, qui s'est tenue du 23 septembre au 28 octobre 1980 à Belgrade, a été marquée par des réflexions sur l'information et la communication. La création d'un Programme international pour le Développement de la Communication (PIDC) a notamment été approuvé par les délégués des 153 Etats présents à Belgrade. Le but de ce Programme est de renforcer les structures des pays en voie de développement en matière d'information et de communication. Tel qu'il est actuellement conçu, ce Programme a également pour objectif l'instauration, après sa définition, du Nouvel Ordre mondial de l'Information, dont I'UNESCO s'est faite le promoteur au cours de ces dernières années, sous l'impulsion notamment de la Commission Mac Bride.

Dans cette optique, la Conférence de Belgrade a du reste adopté deux résolutions visant à renforcer l'influence du rapport Mac Bride, en concrétisant ses conclusions et en préconisant l'élaboration d'une Déclaration sur l'instauration d'un Nouvel Ordre mondial de l'Information et de la Communication.

Dans son rapport sur la Conférence de Belgrade, la délégation de la Suisse adopte une attitude ferme quant au respect des principes de la liberté de presse et d'opinion. Tout en s'associant au consensus sur l'instauration du PIDC, la Suisse s'est vigoureusement opposée aux résolutions concernant les suites à donner au rapport Mac Bride et l'élaboration d'une nouvelle Déclaration. Elle a affirmé son attachement et sa préférence pour des actions concrètes, sousentendant que le rapport Mac Bride et la Déclaration ressortaient de la rhétorique et du débat idéologique. Accentuant même son opposition au consensus, la délégation suisse a été la seule à Belgrade à s'opposer à l'application de la Déclaration sur les médias, visant à stimuler la contribution des organes d'information "à la paix et à la lutte contre le racisme".

L'attitude de la Suisse en matière de communication et d'information reflète une conception "classique" du rôle des médias dans une société ouverte, soumise aux forces du marché, avec ce qu'une telle soumission suppose en termes d'influences, de pressions, voire même d'arbitrages entre courants antagonistes. Il n'est pas étonnant dès lors que la délégation suisse à Belgrade, fidèle en cela à la position habituelle de la Confédération, ait marqué sa préférence pour des actions jugées plus "concrètes", c'est-à-dire la 
contribution des pays riches à l'édification dans les pays en développement d'un système de communication techniquement, économiquement et idéologiquement similaire à celui qui existe dans les pays industrialisés.

Du reste, la Suisse n'a pas manqué de rappeler dans son rapport sur la Conférence de Belgrade qu'elle finançait déjà des projets de développement des médias dans le Tiers Monde par le truchement de la coopération technique et humanitaire et que cette approche lui semblait de loin supérieure à celle prévue par le rapport Mac Bride. On retrouve là une approche caractéristique de la conception officielle de l'aide au développement. Celle-ci semble être conçue comme une stimulation des forces créatrices dans les pays en développement, et non pas comme une contribution au réexamen des rapports de forces au niveau mondial, encore moins à une tentative de réforme de ses rapports internationaux. En effet, l'essentiel du rapport Mac Bride repose sur une analyse des relations entre le Tiers Monde et le monde industrialisé sous I'angle de la communication, et conclut à une situation de domination, à l'instar d'analyses économiques qui sont faites dans les secteurs du commerce Nord-Sud, du transfert de technologie ou des flux financiers mondiaux. Pour les rédacteurs du rapport, le Tiers Monde subit l'ascendant et l'influence du monde industrialisé (avec ses concepts culturels et idéologiques) par le truchement du quasi-monopole dont jouissent les grandes agences de presse et, d'une manière générale, les réseaux de télécommunication (satellites, banques de données, réseaux de télévision et de mondovision). Curieusement, la Suisse ne s'oppose pas à ce concept et en reconnaît même la pertinence. Mais, pour elle, la solution passe par l'appropriation de moyens autonomes de communication, et non pas par une refonte de la notion même de flux d'informations et de son contenu. En bref, pour qu'un pays se libère de la tutelle occidentale, il faut qu'il ait d'abord les moyens techniques à sa disposition.

Reconnaissons la valeur théorique d'une telle conception. La Suède, qui n'est pas coupable de la même mesquinerie en matière d'aide publique au développement, partage cet avis. La SIDA finance en effet des projets de communication dans le Tiers Monde (GuinéeBissau) par le truchement de l'infrastructure d'abord. Sans toujours 
se demander si cette technologie importée à grands frais par le pays aidé a une utilité profonde... Le reproche fondamental qu'on peut opposer à ce concept, c'est qu'il occulte les distorsions profondes de l'actuel système international de communication. II est certes bénéfique d'aider un Etat du Sud à s'équiper (à condition toutefois que cette infrastructure soit réellement adaptée aux possibilités locales et ne soit pas seulement une source de profit pour l'exportateur...) mais un tel effort restera lettre morte si les agences de communication de cet Etat doivent s'intégrer dans un système où leur place est restreinte, où leur voix, pour être entendue, doit s'habiller de concepts, de valeurs, de notions qui leur sont étrangères.

A cet égard, l'attitude suisse est donc restrictive. On a le sentiment une fois de plus que l'aide au développement est appréhendée sous l'angle de la pure rationalité et dans une optique très étroite.

Cela dit, il faut reconnaître que les notions contenues dans le rapport Mac Bride et en filigrane dans les résolutions de Belgrade sont à bien des égards inacceptables pour un éditeur occidental, comme pour le journaliste attaché à des principes séculaires de "liberté" et de "responsabilité". La Suisse en particulier n'accepte pas la notion de "liberté des journalistes" telle qu'elle ressort du rapport final de la Conférence. On se souviendra en outre que la Suisse, comme d'autres pays occidentaux, a rejeté l'idée d'une protection internationale des journalistes, estimant que cette "protection" délivrée par l'Etat hôte camouflait en réalité le désir de contrôler l'activité et la liberté du correspondant. Là encore, ce refus caractérise l'incommunicabilité qui règne actuellement dans les sphère chargées justement d'élaborer un Nouvel Ordre de la Communication! Mais le paradoxe n'est qu'apparent. En réalité, cette situation reflète de profondes divergences d'opinion entre les tenants d'un "ordre journalistique" d'inspiration libérale, dont la Suisse fait partie, et les avocats d'une autre conception de la communication, où à la fois l'organisation et la philosophie du métier d'informer seraient différentes. Rien de très surprenant dans ces conditions que la Suisse manifeste son opposition au projet de Déclaration.

Si la Confédération semble défendre ses acquis en la matière plus 
ardemment que d'autres Etats industrialisés, c'est qu'elle a toujours pris des positions plus tranchées dans une instance culturelle, telle que I'UNESCO, que dans un forum nettement plus politique, comme l'Assemblée générale des Nations Unies, où, par surcroît, elle ne dispose que d'un statut d'observateur. D'autre part, la Suisse, ses représentants officiels au même titre que les délégués des milieux de presse qui participèrent à la Conférence de Belgrade, est très fière de ce qui a été accompli en matière de liberté de presse et de protection des droits du journaliste chez elle. II ne faut pas oublier en effet que la liberté de presse au sens libéral du terme existe en Suisse depuis plus d'un siècle, et que la diversité culturelle, politique, confessionnelle du pays s'est reflétée dans une presse qui, sans être toujours d'une parfaite indépendance, a néanmoins joué son rôle d'aiguillon du pouvoir et de sentinelle de la liberté collective. Il n'est pas aisé dans ces circonstances de remettre en question un tel statut, qui a demandé des années de maturation et qui est aussi enraciné dans les traditions nationales que l'élection au suffrage universel.

Certes, il est vrai que la fragilité du concept de "liberté de la presse et du journaliste" à l'heure des concentrations économiques et du rôle croissant de l'annonceur n'échappe pas à l'auteur de ces lignes. Mais une société, a fortiori la société helvétique si centrifuge, se nourrit parfois de grands principes. Ils sont comme des masques et des phares, tout à la fois. Cachant la réalité, ils aident tout un chacun à avancer dans la nuit.

La Suisse pouvait d'autant moins se rallier à cette Déclaration sur la liberté nouvelle des joumalistes que ce concept est encore très vague, et qu'il ne fait pas l'unanimité chez les nations du Tiers Monde.

Ces remarques, destinées à mettre en perspective l'attitude de la Suisse, ne doivent pas cependant occulter l'essentiel, à savoir la volonté affichée par la Confédération de défendre des acquis dans le domaine des communications mondiales. Si la Suisse, à l'instar des Etats-Unis, part en guerre contre les principes du Nouvel Ordre de l'Information, c'est qu'elle croit y voir l'amorce d'une remise en cause de sa position dominante. A cet égard, il n'est pas inutile de rapprocher cette position intransigeante de celle que défend la Suisse dans d'autres instances des Nations Unies où 
se négocie le Nouvel Ordre Economique International. Dans un cas comme dans l'autre, le "dialogue" Nord-Sud se résume bien souvent à une juxtaposition de principes contradictoires et dont l'issue dépend du rapport de forces de l'instant.

Est-ce à dire que la Suisse n'a rien à offrir au Tiers Monde en matière d'aide au développement de ses moyens de communication ? Certainement pas. La Confédération a réitéré sa politique dans ce domaine, qui est de concourir à cet effort dans le cadre d'accords bilatéraux avec certains pays africains (Mali et Haute-Volta) et dans le cadre de Conventions internationales régissant la distribution des ondes mondiales de télécommunications. Même attitude pour ce qui concerne d'éventuelles facilités accordées au Tiers Monde dans le domaine des tarifs postaux : cette question doit être réglée par une législation internationale.

Pour conclure, mentionnons la querelle opposant le Nord et le Sud, l'Est et l'Ouest sur la question du respect de la souveraineté de l'Etat en matière d'information. Le Tiers Monde reproche aux médias occidentaux de déformer les nouvelles en provenance de chez lui par l'accent qu'ils mettent sur les événements exceptionnels, les conflits internes, les distorsions du pouvoir, etc... A cet appétit pour le spectaculaire, il tente d'opposer le principe d'un flux de nouvelles plus "équilibrées" et qui mettraient en relief les réalisations positives des régimes et des sociétés du Sud. La Suisse, comme d'autres pays industrialisés, y voit poindre l'hydre du contrôle étatique, la mainmise bureaucratique des administrations nationales, la défense des intérêts d'une classe au pouvoir. Bref, la censure. Ce terme tabou de la presse occidentale, cette hantise des salles de rédaction où, pourtant, l'on pratique bien souvent un exercice fort similaire, et qui s'appelle pudiquement "retenue", "respect des institutions établies", ou encore "distanciation par rapport à l'événement".

On ne peut nier bien sûr que dans certains pays du Tiers Monde, comme en Europe au temps des colonels grecs, du fascisme franquiste ou du salazarisme, il existe une pression officielle sur les organes de presse nationaux et internationaux. Plus le pouvoir est concentré dans un Etat, et plus la marge de manœuvre de la presse libre, ou de n'importe quel groupe d'opposants, est réduite : c'est une loi qu'on peut observer partout. Or, il faut bien se ren- 
dre compte que les sociétés tiers-mondistes sont presque toujours organisées sur un modèle unitaire, centralisateur, pour des raisons qu'il ne nous appartient pas d'expliquer ici. C'est une réalité dont il faut tenir compte, comme de la tentation inéluctable qu'elle engendre chez ceux qui en sont les responsables d'en appliquer le principe à tous les secteurs de la vie nationale. Combattre cette réalité au nom d'une autre conception du pouvoir et des relations entre la Cité et les hommes n'a donc pas beaucoup de sens. II conviendrait plutôt d'en accepter l'augure, et d'adapter la vision occidentale de la presse à celle qui prévaut dans le Tiers Monde.

Là encore, on se trouve face à des situations contradictoires : les Etats du Tiers Monde luttent contre tous les fléaux à la fois (sousdéveloppement, manque de cadres, faiblesse des infrastructures, perte d'identité culturelle) alors que le monde industrialisé perçoit la contestation de son rôle dominant et tâche d'y répondre par des arguments qui, par définition, sont rejetés par les autres.

L'essentiel de l'attitude suisse se résume en fin de compte à ceci : les pays en voie de développement souffrent d'un retard considérable dans la course mondiale à l'information. Pour les aider, on peut donner quelques coups de pouce, alléger les frais, améliorer les infrastructures. Mais il n'est pas question de changer les règles de la course. C'est un discours qui n'a que peu de chances d'être compris dans le Tiers Monde où, précisément, on cherche à redéfinir la règle du jeu. 